\title{
Subgrid Scale Model for Finite Difference Simulations of Turbulent Flows in Plane Channels and Annuli
}

\author{
U. SCHUMANN* \\ Institut für Reaktorentwicklung, Gesellschaft für Kernforschung $m b H$, \\ 7500 Karlsruhe, Postfach 3640, Germany
}

Received January 8, 1975

\begin{abstract}
High Reynolds number turbulent flows of incompressible fluids in plane channels and annuli are simulated using a finite difference procedure which integrates the NavierStokes equations in time and in three-dimensional space. This paper describes the finite difference procedure and the subgrid scale (SGS) motion model. The model differs from earlier ones in the following points. The finite difference equations are based on integral conservation equations for each grid volume. As a consequence the SGS stresses are defined as surface mean rather than grid volume mean values of the fluctuating velocity products. This allows us to identify and model the effects of anisotropic grids (especially unequally sided grid volumes) and anisotropic finite difference operators. In this model SGS stresses are split into two parts, one accounting for locally isotropic turbulence, the other for inhomogeneous effects. This results in a model which is meaningful even if the size of the grid volumes is very large. The SGS kinetic energy is calculated using a separate transport equation. The boundary conditions are formulated in a manner consistent with the SGS theory. The method may be used for plane channels and annuli as well, and has been used to simulate flows with up to 65,536 grid volumes. The results agree rather well with experimental values, even for a smaller number of grid volumes.
\end{abstract}

\section{INTRODUCTION}

A finite difference scheme is introduced for direct numerical simulation of turbulent channel flows. This method has been used to simulate high Reynolds number flows of incompressible fluids in plane channels (Poiseuille flow) and concentric annuli of infinite axial lengths, where the mean flow is along the channel axis (see Fig. 1). The flow is assumed to be steady state in its ensemble mean values. The purpose of the method is to obtain detailed turbulent velocity and pressure fields in space and time. These may be used, for example, to investigate turbulence

* On sabbatical leave (1974-1975) at the Advanced Study Program, National Center for Atmospheric Research, Boulder, Colorado, which is sponsored by the National Science Foundation. 


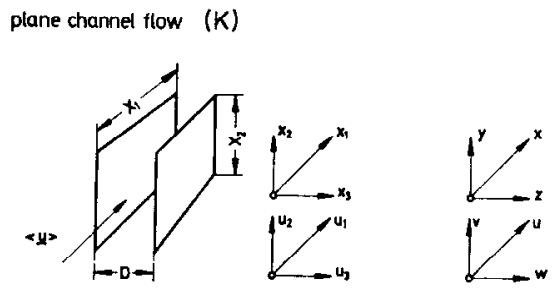

annulus flow $(Z)$
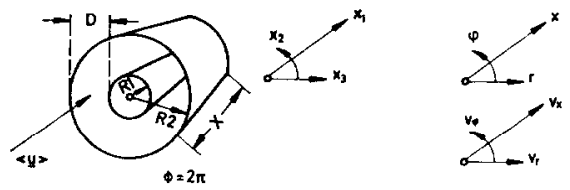

Fig. 1. The channel flows under consideration.

models [20] or wall pressure fluctuations [21]. The present method is an improved form of that described in [19].

The flows are simulated by integrating the three-dimensional and time-dependent Navier-Stokes equations in their primitive form using a finite difference scheme. As known from Schönauer [18] and Galloway and Adler [9], it is impossible with our present computers to obtain turbulent channel flows that agree reasonably well with experiments by using, e.g., a finite difference form of the Navier-Stokes equations in a purely formal sense. The reason is that for resolution of all important scales of turbulence at Reynolds number $\operatorname{Re}_{m}$ of $10^{5}$, at least $10^{9}$ grid volumes are needed, whereas the number of grid volumes feasible today is of the order $10^{5}$ [19]. So the formal finite difference scheme has to be completed with models based on physical reasoning that accounts for those scales of turbulence not resolvable by the limited number of grid volumes. This was the approach first used by Smagorinsky [22] and Lilly [15] and successfully employed by Deardorff [4-7]. In that approach, the Navier-Stokes equations are averaged over volumes comparable in size to the grid volumes of the finite difference scheme, in order to filter out the subgrid scale (SGS) motions. In this way only the gross scale turbulence is simulated directly. The momentum transport by the fine scale or SGS motions is described by an eddy viscosity model. In principle, the SGS motions and, consequently, the theory and its deficiencies cancel out if we approach an infinitely fine grid system. This distinguishes the "direct numerical simulation method" being used here from the common "transport models" which start from the Navier-Stokes equations averaged over ensembles or time. Moreover it can be 
claimed that such models for the SGS motions are more universally valid than for the total turbulence, since they are much less dependent on channel geometry.

Until now, the only flow geometry that has been considered using this type of method is the plane channel or, in a geometrically similar case, the planetary boundary layer. To show the independence of geometry for some practically used turbulence models, at least one additional geometry should be investigated. Due to its technical importance, e.g., in nuclear engineering, the annular flow is of interest in this connection. Therefore, some work had been started to apply Deardorff's method to annular flow, using cylindrical coordinates. Of help here, as far as the finite difference procedure away from boundaries is concerned, is the work done by Williams [24].

But the existing SGS models have been found unsuitable for cylindrical coordinate systems. Here we are confronted with the problem of using an anisotropic grid in that the ratio of side lengths of grid volumes varies with the radial coordinatc. The need for some theory to acocunt for unequal side lengths of the grid volumes has also been recognized for Cartesian coordinates [7]. It is one of the main purposes of this paper to describe such a theory. For brevity, we present it for Cartesian coordinates only. The second major problem encountered concerns the effects of inhomogeneities. The older theories assume locally isotropic turbulence. This causes rather large errors, especially near the walls, as can be seen from the results obtained by Deardorff [4]. In the following pages, the new method will be described and some results will be reported which demonstrate its usefulness.

\section{The Averaged Basic Equations}

We start from the basic equations describing conservation of mass and momentum for incompressible flows.

$$
\begin{gathered}
\operatorname{div} \mathbf{u}=\partial u_{j} / \partial x_{j}=0 \\
\frac{D u_{i}}{D t} \equiv \frac{\partial u_{i}}{\partial t}+\frac{\partial}{\partial x_{j}}\left(u_{j} u_{i}\right)=-\frac{\partial p}{\partial x_{i}}+\frac{1}{\operatorname{Re}} \frac{\partial^{2} u_{i}}{\partial x_{j}{ }^{2}}+P_{x} \delta_{1 i}, \quad i=1,2,3
\end{gathered}
$$

All velocities $u_{i}$, coordinates $x_{i}$ (see Fig. 1), and the time $t$ are made dimensionless by means of the length scale $\hat{D}$ separating the walls, by the friction velocity $\hat{u}_{0}=\left(\hat{\tau}_{0} / \hat{\rho}_{0}\right)^{1 / 2}$, and by the time scale $\hat{D} / \hat{u}_{0}$. The quantity $\hat{\tau}_{0}$ is the time mean wall stress averaged over both walls so that $\hat{\tau}_{0}=\left(\hat{P}_{x} \hat{D}\right) / 2$; here $\hat{P}_{x}$ is the dimensional driving force per unit volume in the axial direction which can be interpreted as 
the time mean kinematic pressure gradient. $p$ is the dimensionless fluctuating kinematic pressure. $\operatorname{Re}=1 / v$ is a Reynolds number

$$
\operatorname{Re}=\hat{u}_{0} \hat{D} / \hat{\nu}
$$

where $\hat{v}$ is a constant kinematic viscosity. This Reynolds number is different from the usual Reynolds number $\operatorname{Re}_{m}$ based on the mean axial velocity $\hat{u}_{1 i m}$ :

$$
\operatorname{Re}_{m} \equiv \hat{u}_{1 m} \hat{D} / \hat{\nu}=u_{1 m} \operatorname{Re}, \quad u_{1 m}=\hat{u}_{1 m} / \hat{u}_{0} .
$$

From definition of the dimensional units, the dimensionless driving force is $P_{x}=2$. The summation convention applies to all subscripts.

In order to generate finite difference formulas from these equations we average them over small volumes as defined by our grid (see Fig. 2). Any quantity $y$ averaged over such a volume $V$ is denoted by $V \bar{y}$.

$$
v_{\vec{y}} \equiv \frac{1}{\Delta x_{1} \Delta x_{2} \Delta x_{3}} \int_{\Delta x_{1}} \int_{\Delta x_{2}} \int_{\Delta x_{3}} y\left(z_{1}, z_{2}, z_{3}\right) d z_{1} d z_{2} d z_{3} .
$$

Note that the volume over which the average is taken is fixed in space, so that $\nu_{\bar{y}}$ is defined only on discrete points. Then we integrate by parts using Gauss' theorem.

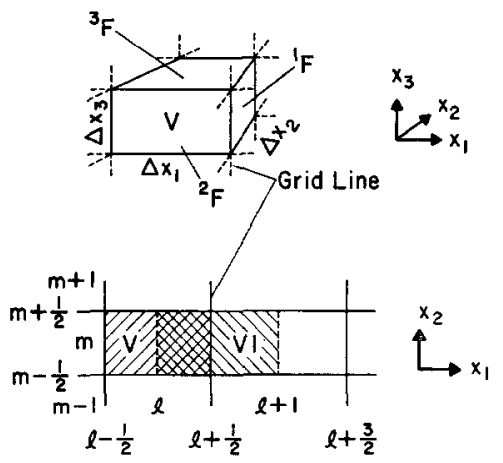

FIG. 2. Grid volumes and surfaces.

This results in a relationship between the time derivative of the physical quantity averaged over that volume and its transport across the surface of that volume in terms of surface averages. We denote the surface area of a grid volume, the normal of which is in the $j$-coordinate direction, by ${ }^{j} F$ (presuperscripts are introduced for those indices for which the summation convention does not apply). The mean value of any quantity $y$ averaged over ${ }^{j} F$ is denoted by ${ }^{j} \bar{y}$, e.g.:

$$
\stackrel{1-}{y} \equiv \frac{1}{\Delta x_{2} \Delta x_{3}} \int_{\Delta x_{2}} \int_{\Delta x_{3}} y\left(x_{1}, z_{2}, z_{3}\right) d z_{2} d z_{3} \text {. }
$$


Integration by parts gives us relationships of the form

$$
\frac{v-\frac{\partial}{\partial x_{1}} y}{\Delta x_{1}}\left\{\frac{1}{y}\left(x_{1}+\frac{\Delta x_{1}}{2}\right)-{ }^{1-} y\left(x_{1}-\frac{\Delta x_{1}}{2}\right)\right\} \equiv \delta_{1}{ }^{1-} \bar{y}
$$

Here, $\delta_{j}$ is the usual finite difference operator [24].

Applying these definitions to Eq. (1), the conservation law of mass, results in

$$
\overline{\operatorname{div} \mathbf{u}}=\delta_{j} \overline{u_{j}}=0 .
$$

Now, let ${ }^{j} \bar{u}_{j}$ be equal to the velocity component in the $j$-direction defined in a staggered grid system (see Fig. 3 ). Then we see that we do not need any approximation for this equation. This seems to be the reason why staggered grids are used so often in hydrodynamics.

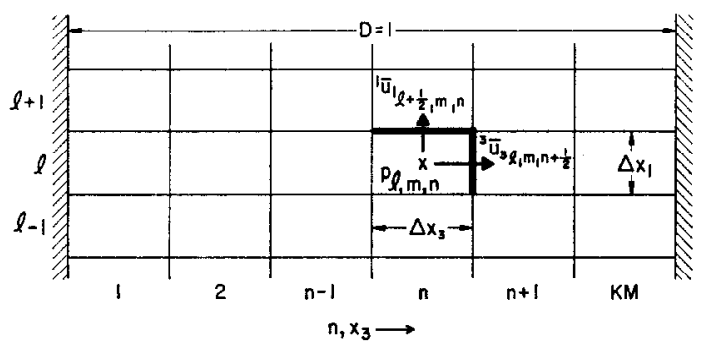

FIG. 3. Staggered grid.

In order to get the averaged conservation law for the different velocity components, we average Eq. (2) over volumes $V i$ which are of the same size and form as the volume $V$, but shifted so as to surround the position of the velocity component within the staggered grid. Any quantity $y$ averaged over such a volume is denoted by ${ }^{V i} \bar{y}$, where $i$ corresponds to the coordinate direction in which the volume was shifted. Then we get

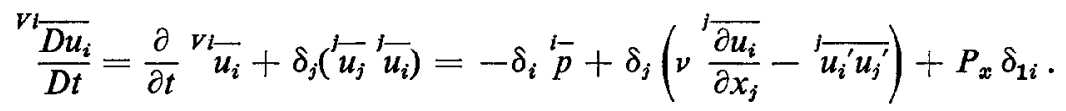

The terms

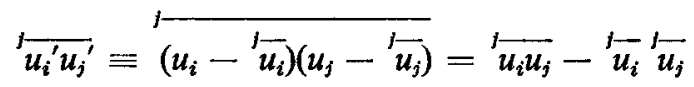

are the SGS Reynolds stresses by which the effect of the fine-scale motion is identified. Here and below the summation convention refers to all lower subscripts on the right not enclosed in square brackets. Other indices of equal name take the corresponding values. 
So far no approximations have been involved. Indeed, the equations above are the conservation laws written in their integral form for grid volumes. We might have started from them right away, but as they do not exist in general for grid volumes in curvilinear coordinate systems, we prefer to start from the basic equations in their differential form.

The "volume balance procedure" used above is of course quite usual in continuum mechanics. Nevertheless, this approach is different from the "filter procedure" used in the past by Lilly [15] and Deardorff [4-7]. They average over a volume which is not fixed in space. This averaging procedure is equivalent to a convolution of the original field with a filter function, as shown by Leonard [13]. This excludes the possibility of integration by part. Therefore, the older theories speak of volume-averaged SGS stresses, which is physically unrealistic in the finite difference approach, since the SGS stresses are basically surface forces. Those theories have, therefore, not been able to describe the effects of grid anisotropy. One drawback of the "volume balance procedurc" is, of course, that we need quite a strange notation in order to distinguish between the different types of averages which appear (see summary of operator notation).

Approximations are needed for many terms appearing in the averaged conservation law of momentum Eq. (9). There are two types of unknown quantities: the SGS stresses $\overline{u_{i}{ }^{\prime} u_{j}{ }^{\prime}}$ and those averaged quantities which must be known at positions not coinciding with the positions of the corresponding variables in the staggered grid. The latter unknown quantities can be evaluated by the usual difference formulas from the corresponding staggered grid values in their neighborhood. A second-order energy-conserving scheme is used. The resultant finite difference equations are the same as those used by Deardorff [4] for Cartesian and by Williams [24] for cylindrical coordinates, as far as the gross scale velocities are concerned. Therefore, volume balance and filter averaging procedures give the same result in this respect. The approximations used for the SGS stresses form the topics of the next section.

\section{THE SGS EdDY Viscosity ModeL}

\section{Splitting into "Locally Isotropic" and "Inhomogeneous" Parts}

We look for an approximate equation to correlate the SGS Reynolds stresses ${ }^{j} \overline{u_{i}{ }^{\prime} u_{j}{ }^{\prime}}$, as defined by Eq. (10), with the gross scale variables. Although we are not able to model these terms without any empirical adjustment, we should use as little experimental support as possible. Then we might be able to extract new empirical information and to cover a wide range of boundary conditions.

It is well known [17] that any turbulent flow is locally isotropic and possesses 
an inertial subrange if the Reynolds number is sufficiently high. From measurements of velocity spectra we may identify that characteristic length scale which separates the anisotropic part from the locally isotropic part. Using the results of Laufer [12] and Comte-Bellot [3] has lead to the finding that the grid cell size

$$
h=\left(\Delta x_{1} \Delta x_{2} \Delta x_{3}\right)^{1 / 3}
$$

falls within the local isotropic range if the Reynolds number $\operatorname{Re}_{x}=\hat{x}_{3} \hat{u}_{0} / \hat{v}$ defined by the distance of the wall $\hat{x}_{3}$ is larger than 200 . Taking $\hat{x}_{3}=h \hat{D}$ and $\hat{u}_{0} \approx \hat{u}_{1 m} / 30$ [3] (see Eq. (4)), we find that for a grid side length $h$ of the order $D / 30$, and for a Reynolds number $\operatorname{Re}_{m}>2 \times 10^{5}$, the assumption of local isotropy is justified except for the wall-adjacent grid cells.

In the inertial subrange, the energy spectrum $E(k)$ as a function of the wavenumber $k$ (for its definition, see Hinze [10, Eq. 3-57]) is equal to the so-called Kolmogorov spectrum [11]:

$$
E(k)=\alpha\langle\epsilon\rangle^{2 / 3} k^{-5 / 3}
$$

Due to the experimental results obtained by several workers (some are compiled in [19]), we may assume "Kolmogorov's constant $\alpha$ " to be

$$
\alpha=1.5 \text {. }
$$

The dissipation rate $\epsilon$ is defined by

$$
\epsilon \equiv \nu \frac{\partial u_{i}}{\partial x_{j}}\left(\frac{\partial u_{i}}{\partial x_{j}}+\frac{\partial u_{j}}{\partial x_{i}}\right)
$$

The brackets $\langle>$ denote the time mean value. From the experimental results of Comte-Bellot [3] we find that the range where (12) is valid usually extends to even larger scales than the local isotropic subrange.

On the other hand, the requirement of $h<D / 30$ (or even more, $h \ll D / 30$ ) is still quite restrictive in view of our present computer resources. Therefore we are looking for a way to separate the SGS stresses into one part which can be evaluated using the assumption of local isotropy and a second part which accounts for the inhomogeneities especially in the near-wall region. There, we refer to Prandtl's mixing length model and the resulting logarithmic "law of the wall" $[10,17]$ : both are known to be quite suitable for the near-wall region. In order to keep the theory simple, we adopt an eddy viscosity concept (Boussinesq approximation [17]) to relate the stresses to the strain tensor $D_{i j}$ respectively to its finite difference analog $\bar{D}_{i j}$.

$$
D_{i j}=\frac{\partial u_{i}}{\partial x_{j}}+\frac{\partial u_{j}}{\partial x_{i}}, \quad \bar{D}_{i j}=\delta_{j} \stackrel{i}{u_{i}}+\delta_{i} \stackrel{i}{u_{j}}
$$


We therefore write

$$
\overline{u_{i}^{\prime} u_{j}^{\prime}}=-\underbrace{{ }^{i j} \mu\left(\bar{D}_{i j}-\left\langle\bar{D}_{i j}\right\rangle\right)}_{\text {"locally isotropic part" }} \underbrace{-{ }^{i j} \mu *\left\langle\bar{D}_{i j}\right\rangle}_{\text {"inhomogeneous part" }}+\frac{1}{3} \delta_{i j} \stackrel{k}{u_{k}^{\prime} u_{k}^{\prime}}
$$

The coefficients ${ }^{i j} \mu$ and ${ }^{i j} \mu *$ arc eddy viscosities accounting for locally isotropic and inhomogeneous turbulence, respectively. The locally isotropic part of the SGS stresses is set proportional to the fluctuating part of the strain tensor in order to get zero time mean values of the SGS stresses for $i \neq j$. As these stress components are zero in isotropic turbulence (by definition), this part of the model is thus made consistent with the assumption on which it is based. The channel turbulence is inhomogeneous due to the nonzero components of the time mean strain. This is reflected by the inhomogeneous part of the above assumption. The last term in Eq. (16) has been added to ensure that the value of $\delta_{i j} \bar{u}_{i}^{\prime} u_{j}^{\prime}$ is equal for both sides of the equation [10, p. 21]. This term can be added to the pressure, resulting in a virtual pressure $\bar{p}={ }^{j} \bar{p}+\frac{1}{3} \overline{u_{k}{ }^{\prime} u_{k}^{\prime}}$ (independent of $j$ ). Actually, $\bar{p}$ is that pressure that is simulated by the finite difference scheme. We should note that ${ }^{i j} \mu$ and ${ }^{i j} \mu^{*}$ are not tensors. The dependence of $i$ and $j$ is introduced solely by the anisotropy of the finite difference grid. In the following sections we describe the models used for these eddy viscosities.

\section{Locally Isotropic SGS Eddy Viscosity}

The SGS eddy viscosity ${ }^{i j} \mu$ is modeled as

$$
{ }^{i j} \mu=c_{2}\left({ }^{j} F^{j} \overline{E^{\prime}}\right)^{1 / 2}{ }^{i j} c \text {. }
$$

This is an analog to the Prandtl-Kolmogorov proposal for the total eddy viscosity [17, Eq. (3.117)]. The characteristic length scale is taken from ${ }^{j} F$ as this is the size of the surface over which we are averaging. If the grid is refined, as ${ }^{j} F$ approaches zero the SGS stresses become zero by definition (see Eq. (10)). Thus, this property is retained in this model. In earlier proposals [4-7, 15], $h$ (Eq. (11)) has been taken as the characteristic length scale, which is a measure for the grid cell volume and thus is not consistent with the fact that the stresses are basically surface forces. The characteristic velocity is the root value of the SGS kinetic energy contained within the area ${ }^{j} \mathrm{~F}$ :

$$
\overline{E^{\prime}} \equiv \frac{1}{2} \overline{\left(u_{i}-\overline{u_{i}}\right)^{2}}=\frac{1}{2}\left[\overline{u_{i}^{2}}-\left(\overline{u_{i}}\right)^{2}\right]
$$

We evaluate ${ }^{j} \overline{E^{\prime}}$ from

$$
{ }^{\prime} \overline{E^{\prime}} \approx{ }^{j} c_{5}{ }^{v} \overline{E^{\prime}},
$$


where

$$
{ }^{j} c_{5} \equiv \frac{\left\langle\overline{E^{\prime}}\right\rangle}{\left\langle\overline{E^{\prime}}\right\rangle}
$$

and

$$
v_{\overline{E^{\prime}}} \equiv \frac{1}{2} \overline{\left(u_{i}-V_{\overline{u_{i}}}\right)^{2}}=\frac{1}{2}\left(\overline{u_{i}{ }^{2}}-\left(V_{\overline{u_{i}}}\right)^{2}\right)
$$

is the SGS kinetic energy. For the determination of ${ }^{j} c_{5}$, see below. The SGS kinetic energy is determined from a separate transport equation as described in Section IV. The coefficient ${ }^{i j} c$ is introduced to account for anisotropy introduced by finite differencing: the definition of $\overline{u_{i}^{\prime} u_{j}^{\prime}}$ does not allow these stresses to be dependent upon the grid spacings $\Delta x_{j}$. On the other hand, $\bar{D}_{i j}$ is dependent upon $\Delta x_{j}$. Therefore this coefficient shall compensate for the dependence. The need for such a compensation and the evaluation of ${ }^{i j} \mathrm{c}$ is described further in Section IV.

The coefficients ${ }^{j} c_{5}$ and ${ }^{i j} c$ are both of the order of one. They would be absorbed into $c_{2}$ if we are to neglect geometrical details of the averaging and finite difference operator as has been done in older SGS theories. The constant $c_{2}$, however, must be determined so that the energy dissipation due to the eddy viscosity in the simulated gross-scale flow is of the same size as the viscous dissipation which represents the energy sink in reality. To formulate this requirement quantitatively we need the conservation law for the SGS kinetic energy ${ }^{V} \overline{E^{\prime}}$.

We get the conservation law for this quantity by evaluating the time differential of the definition, Eq. (21), using Eqs. (1), (2), (8), and (9). In order to simplify the expressions we use the identity

$$
\delta_{i}(a b)=\bar{a}^{i} \delta_{i} b+\bar{b}^{i} \delta_{i} a
$$

where $\bar{y}^{i}$ is the arithmetic mean value of any quantity $y$ at two positions separated by one grid interval in the $x_{i}$-direction. We also use one approximate equation,

$$
\nu_{\overline{u_{i}}} \approx \overline{\bar{u}_{i}}
$$

which can be defended using the mean value theorem of the integral calculus so as to be valid up to errors of the order $O\left(\Delta x^{2}\right)$. The result: 


$$
\begin{aligned}
& \frac{\partial}{\partial t} v_{\overline{E^{\prime}}}+\underbrace{\delta_{j}\left(\bar{u}_{j}^{\prime} \overline{E^{\prime}}\right)}_{\text {convection }}=-\underbrace{\overline{\bar{u}_{i}^{\prime} u_{j}^{\prime}} \delta_{j}^{\prime} \overline{u_{i}}}_{\text {production }}-\underbrace{v_{-}^{\epsilon}}_{\text {dissipa tion }}+\underbrace{\nu\left\{\left(\delta_{j}^{j} \overline{u_{i}}\right)\left(\delta_{j}^{\prime} \overline{u_{i}}+\delta_{i} \overline{u_{j}}\right)\right.}_{\text {viscous gross scale dissipation }}\}
\end{aligned}
$$

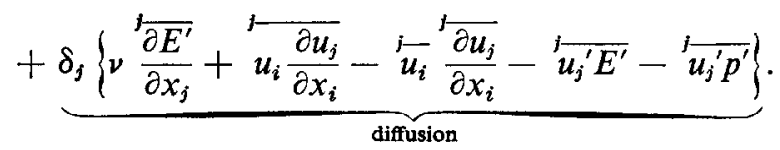

The coefficient $c_{2}$ can be found by introducing Eqs. (16) (neglecting the inhomogeneous part due to the assumption of local isotropy) and (17) into Eq. (24) and taking the time mean value. In locally isotropic turbulence the time mean values of the convection and diffusion terms vanish. To simplify the resulting equations, we neglect the viscous gross-scale dissipation rate and assume that the SGS kinetic energy and the square strain of the gross-scale motion are uncorrelated. Both assumptions can be avoided [19] but they are of minor importance. The result is the following equation for $c_{2}$.

$$
\left.c_{2}=\langle\epsilon\rangle /\left[\left\langle{ }^{j} F^{j} \overline{E^{\prime}}\right\rangle^{1 / 2}\left\langle\overline{\left\langle\delta_{i}{ }^{j-}{ }_{j}+\delta_{j}{ }^{i-}{ }^{\prime}\right.}{ }_{i}\right) \delta_{j}{ }^{j-}{ }_{u_{i}}^{i j} c\right\rangle\right]
$$

All correlations involved in this equation and in Eq. (20) can be evaluated in a purely formal way without any further assumptions (see below, however). This can be done once for all integration steps because they depend on the grid system only. The mathematical procedure is to some extent similar to that used by Lilly [14]. It has been extended to account for unequal grid side lenghts and the definition of the different averages occurring. Moreover, Lilly's method was rather expensive, because he had to evaluate by numerical means some integral expressions with up to six independent variables. Using a formulation provided mainly by Uberoi and Kovasznay [23], we can reduce this number to half, which reduces computing time for these integrals from hours to seconds. However, since we must evaluate a large number of these integrals, this would still be too expensive. Therefore, some approximate equations were developed to fit the numerical results of the integrals. For details, see the Appendix. Some typical results are reported in Table I.

The principal way to determine the constant $c_{2}$, using the assumption of local isotropy and Kolmogorov's spectrum (Eqs. (12), (13)), is most simply demonstrated by employing Lilly's [15] approximate method to evaluate the terms appearing in Eq. (25):

$$
\begin{aligned}
& \left\langle\overline{E^{\prime}}\right\rangle \approx \int_{\pi / n}^{\infty} E(k) d k, \\
& \left\langle{\overline{\left(\delta_{i}{ }^{\prime} u_{j}+\delta_{j}{ }^{l-u_{i}}\right)}}^{j} \delta_{j}{ }^{\prime} \vec{u}_{i}\right\rangle \approx 2 \int_{0}^{\pi / h} k^{2} E(k) d k, \\
& \left.{ }^{j} F\right)^{1 / 2} \approx h, \quad{ }^{i j} c \approx 1 \text {. }
\end{aligned}
$$


TABLE I

Coefficients Appearing in the SGS-Theory

\begin{tabular}{l|l|l}
\hline & \multicolumn{2}{|c}{$\Delta x_{1}: \Delta x_{2}: \Delta x_{3}$} \\
\cline { 2 - 3 } Factor & $=1: 1: 1$ & $=0.125: 0.05: 005$ \\
\hline$c_{2}{ }^{a}$ & 0.143 & 0.141 \\
${ }^{1} c_{5}$ & 0.828 & 0.614 \\
${ }^{2} c_{5}$ & 0.828 & 0.915 \\
${ }^{8} c_{5}$ & 0.828 & 0.915 \\
$c_{3}$ & 1.02 & 0.884 \\
$c_{4} / c_{2}$ & 1.63 & 1.57 \\
\hline
\end{tabular}

a The value used in the computations is smaller by a factor of $\frac{1}{3}$.

From Eqs. (12) and (13) we then get

$$
c_{2} \approx(2 / 3 \alpha)^{3 / 2} \simeq 0.094
$$

This has to be compared with the result given in Table I.

It must be stated, however, that the predicted value of $c_{2}$ determining the size of the fluctuating part of the SGS stresses, which by theory depends on Kolmogorov's constant only, was found to be too high for reasonable results. It caused too-large dampings and had to be decreased in an empirical way. A similar effect has been found by Deardorff $[4,5]$. The value of $c_{2}$ used in the final computation was smaller by a factor of $\frac{1}{3}$ than that found theoretically. This factor gave reasonable results for all grids and both channel types (plane and annular) considered, which represents some progress in comparison to [19], where different factors had to be used for plane and annular channels; this is due to the correction factor ${ }^{i j} c$ in Eq. (17), which has not been used in the older version of this method. We expect the explanation for the difference between theory and experiment to be connected with finite difference errors. It seems that while the finite difference scheme is energy conserving, the actual energy transfer toward larger wavenumbers in the gross-scale simulation is too large, so that we get larger values of $\bar{D}_{i j}^{2}$ than predicted by theory. Higher-order difference schemes might be useful, therefore. On the other hand, the stencils used in higher-order schemes usually include grid points which are separated by more grid intervals than those used in a second-order scheme. For the error analysis we have to assume that the large-scale flow is sufficiently smooth and correlated in a deterministic manner even for these larger 
intervals. The correlation between the velocities at two points separated by a distance $r$ decreases proportional to $r^{2 / 3}$ for isotropic turbulence with Kolmogorov's spectrum (see Appendix, Eq. (A-2)). It is therefore not a priori clear whether the gain in accuracy using a higher-order scheme is large enough to justify the increased computation time. Moreover, it follows from Eqs. (12), (17), and (26) that the SGS eddy viscosity ${ }^{i j} \mu$ is of the order $h^{4 / 3}$, and any error in the model must be assumed to be of the same order. As long as the eddy viscosity is larger than the molecular viscosity, this is the dominant error even for a second-order finite difference scheme. Here, we use the second-order scheme for simplicity.

\section{Inhomogeneous SGS Eddy Viscosity}

The inhomogeneity of the flow would have negligible effects on the SGS momentum transport if the size of the grid surfaces ${ }^{j} F$ were extremely small. However, this limit cannot be accomplished, especially in the near-wall region. Here the characteristic eddy length scale, the mixing length $L$, becomes smaller than the root value of ${ }^{j} F$. If $\left({ }^{j} F\right)^{1 / 2}$ is much larger than $L$, all momentum is transported by the SGS motion and the gross-scale momentum transport becomes negligible. Thus, we have the limit

$$
\lim _{j_{F \rightarrow \infty}} \overline{u_{i}^{\prime} u_{j}^{\prime}}=\left\langle u_{i}^{\prime} u_{j}^{\prime}\right\rangle
$$

The right-hand side represents the common time mean Reynolds stresses. Therefore, we model the inhomogeneous part of the SGS momentum transport so that it becomes equal to common models for the time mean Reynolds stresses in this limiting case. The simplest model in this respect is Prandtl's mixing length model, which is known to be valid in the near-wall region. If, however, the value of $\left({ }^{j} F\right)^{1 / 2}$ is small in comparison to the mixing length, then the former becomes the characteristic length scale. For the channel flows under consideration here, the eddy viscosity ${ }^{i j} \mu^{*}$ must be known only for the subscripts $i=1, j=3$, because then either the other stress components or their gradients used in the finite difference equations are zero. The only factor of interest, ${ }^{13} \mu^{*}$, is calculated by means of the equations

$$
\begin{aligned}
{ }^{13} \mu^{*} & =\left({ }^{3} L\right)^{2}\left|\delta_{3}\left\langle u_{i}\right\rangle\right|, \\
\left({ }^{3} L\right)^{2} & =\min \left(c_{10}{ }^{3} F, L^{2}\right), \\
L & =\kappa x_{3}{ }^{\prime} .
\end{aligned}
$$

Equation (31) is the mixing length model $[10,17]$. The factor $\kappa$ is Karman's constant $(\kappa \approx 0.4)[10,17]$ and $x_{3}{ }^{\prime}$ is the distance to the nearest wall. The factor $c_{10}$ may be estimated on the basis of typical values of $L^{2}$ and a size of ${ }^{3} F$ just large 
enough to make the mean value of $\left(u_{1}^{\prime} u_{3}{ }^{\prime}\right)$ taken over this area equal to its time mean value (Eq. 30) with negligible error only. Using the measurements of Comte-Bellot [3], especially those of the correlations in the $x_{1}$ - and $x_{3}$-directions, to estimate the size of ${ }^{3} F$ in this special case, we may support the assumption

$$
c_{10} \approx 0.01 \text {. }
$$

Equation (33) can be extended, according to Van Driest [8], to account for low local Reynolds numbers [19]. But this is of small importance for those Reynolds numbers $\left(\operatorname{Re}_{m}=3 \times 10^{5}\right)$ and grid spacings (Table II) actually used.

TABLE II

Specifications of Cases $K 1-K 4, Z 1-Z 4, K 3^{+}, K 4^{+}, Z 3^{+}$

\begin{tabular}{lllllllll}
\hline & $K 1$ & $K 2$ & $K 3 / K 3^{+}$ & $K 4 / K 4^{+}$ & $Z 1$ & $Z 2$ & $Z 3 / Z 3^{+}$ & \multicolumn{1}{c}{$Z 4$} \\
\hline$\Delta x_{1} / D$ & 0.125 & 0.125 & 0.0625 & 0.0625 & 0.25 & 0.25 & 0.125 & 0.125 \\
$\Delta x_{2} / D$ & 0.125 & 0.125 & 0.0625 & 0.0625 & $\pi / 16$ & $\pi / 16$ & $\pi / 32$ & $\pi / 32$ \\
$\Delta x_{3} / D$ & 0.0625 & 0.0625 & 0.0625 & $0.018 / 0.042$ & 0.0625 & 0.0625 & 0.0625 & 0.018 to 0.042 \\
$X_{1} / D$ & 2 & 4 & 4 & 4 & 2 & 4 & 4 & 4 \\
$X_{2} / D$ & 1 & 2 & 2 & 2 & $\pi$ & $2 \pi$ & $2 \pi$ & $2 \pi$ \\
$\begin{array}{c}\text { Number of } \\
\text { grid cells }\end{array}$ & 2048 & 8192 & 32768 & 65536 & 2048 & 8192 & 32768 & 65536 \\
$\begin{array}{c}\text { Problem-time } \\
\text { per } \hat{D} / \hat{u}_{0}\end{array}$ & 5.6 & 3.8 & $0.9 / 3.98$ & $+0.2 /+0.6^{a}$ & 7.4 & 12.3 & $1.4 / 4.87$ & $+0.44^{a}$ \\
$\begin{array}{c}\text { Computing } \\
\text { time IBM }\end{array}$ & $35^{\prime}$ & $2 \mathrm{~h} 30^{\prime}$ & $6 \mathrm{~h} / 20 \mathrm{~h}$ & $+3 \mathrm{~h} /+14 \mathrm{~h}^{a}$ & $40^{\prime}$ & $5 \mathrm{~h} 30^{\prime}$ & $7 \mathrm{~h} 40^{\prime} / 25 \mathrm{~h}$ & $+7 \mathrm{~h}^{a}$ \\
$370 / 165$ & & & & & & & & \\
\hline
\end{tabular}

${ }^{a}$ Starting from initial values which are obtained by interpolating final results of $K 3 / Z 3$.

We may assume that the finite difference grid is dense enough to resolve the gradient $\delta_{3}\left\langle u_{1}\right\rangle$ of the time mean velocity field in the interior of the flow field. Therefore, the gradients are independent of $\Delta x_{3}$ and the correction factor ${ }^{i j} c$ introduced in Eq. (17) is not necessary here. (Special considerations are necessary at the walls; see Section V).

An important practical problem connected with this model is to evaluate the time mean values needed in the model and later on in extracting statistical results. Because of limited computer time we get results for rather short problem times. 
So the data basis is not large enough to compute real time mean values. Moreover, we need what is denoted by \langle\rangle already during the transient part of integration. Therefore, these mean values are calculated here as space mean values taken for those planes which are parallel to the walls. For sufficiently large planes these values and the time mean values are the same for steady state $[17, \mathrm{p} .21]$.

\section{SGS Kinetic ENERgy TRANSPORT EQuation}

The SGS kinetic energy ${ }^{V} \overline{E^{\prime}}$ as defined by Eq. (21) is calculated by means of a separate transport equation deduced from its conservation law, Eq. (24). Here, the unknown turbulence terms are modeled in the manner suggested by Lilly [15].

$$
\frac{\partial}{\partial t} V_{\overline{E^{\prime}}}+\delta_{j}\left({ }^{j} c_{5}{ }^{j} \bar{u}_{j}{ }^{V} \overline{E^{\prime}}\right)=P-c_{3}\left({ }^{\nu} \overline{E^{\prime}}\right)^{3 / 2} / h+\delta_{j}\left[\left(\nu+c_{7} c_{2}\left({ }^{j} F^{j} c_{5}{ }^{V} \overline{E^{\prime}}\right)^{1 / 2}\right) \delta_{j}{ }^{\nu} \overline{E^{\prime}}\right] .
$$

Here, $P$ is the production term, which is the consequence of Eqs. (16) and (17) and the assumption of local isotropy:

$$
P=c_{4} h^{i j} c \bar{D}_{i j}^{2}\left({ }^{\nu} \overline{E^{\prime}}\right)^{1 / 2} .
$$

The length scale $h$ is that defined in Eq. (11). The quantity $\bar{D}_{i j}^{2}$ stands for any specific finite difference form of the strain tensor squared. The square value of $\bar{D}_{i j}$ as defined by Eq. (15) is not appropriate as this is not defined for the midpoint of a grid volume. The formula finally used is

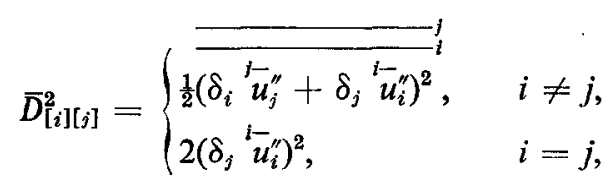

corresponding to the formula used by Deardorff [5]. However, in this case we use fluctuating velocities instead of total velocities. This is consistent with the assumption of local isotropy and results in zero production, if the flow becomes laminar. The constants ${ }^{j} c_{5}$ are those given in Eq. (20). The factor $c_{4}$ is found from the condition that the time mean value of $P$ is equal to the time mean value of the exact production term as defined in Eq. (24). If we neglect the impacts of the finite differencing, ${ }^{j} c_{5}$ would be equal to one and $c_{4}$ equal to $c_{2}$, as given by Eq. (25). $c_{7}$ corresponds to a turbulent Prandtl number describing the ratio of the eddy diffusivities for SGS energy and momentum transport. It was arbitrarily fixed at $c_{7}=0.3$. The results have been found to be insensitive to this value. The factor $c_{3}$ can be evaluated approximately, using Eq. (12): 


$$
c_{3} \approx\langle\epsilon\rangle h /\left\langle^{V} \overline{E^{\prime}}\right\rangle^{3 / 2} \approx\langle\epsilon\rangle h /\left[\int_{\pi / h}^{\infty} E(k) d k\right]^{3 / 2}=\pi\left(\frac{2}{3 \alpha}\right)^{2 / 3} .
$$

Actually, however, ${ }^{i} c_{5}, c_{4}$, and $c_{3}$ have been evaluated, with all geometrical details accounted for in the same manner as described for the factor $c_{2}$.

The factors ${ }^{i j} c$ appear due to Eq. (17). If ${ }^{i j} c$ is set to one everywhere, it has been found that for strongly anisotropic grids with, e.g., $\Delta x_{1} \gg \Delta x_{2} \approx \Delta x_{3}$, the resultant velocity field does not have the nearly random structure expected for turbulent flows. Instead, the fluctuating velocity field becomes nearly independent of the $x_{2}$ and $x_{3}$ directions. This effect can be explained as due to the large differences between the magnitudes of the single values $\bar{D}_{[i][j]}^{2}$ contributing to the production term $P \sim \bar{D}_{2 j}^{2}$. In the inertial subrange $\left\langle\bar{D}_{[i][j]}^{2}\right\rangle$ is proportional to $\Delta x_{[j]}^{-4 / 3}$, so each variation of the velocity field in the $x_{2}$ or $x_{3}$ direction results in much larger contributions to the production term than do such variations in the $x_{1}$ direction; this generates large SGS kinetic energy and thus large eddy viscosity ${ }^{i j} \mu$ (Eq. (17)) in this region, damping out every velocity fluctuation. Therefore, the anisotropic form of the grid affects anisotropic values of $\left\langle\bar{D}_{[i][i]}^{2}\right\rangle$ which in turn cause a nonphysical anisotropy of the velocity field. This is even enforced by the anisotropic form of the finite difference model as given by Eq. (37). The solution of this problem appears to be a correction factor

$$
{ }^{i j} c=\left(\left\langle\bar{D}_{i j}^{2}\right\rangle \mid\left\langle\bar{D}_{[i][j]}^{2}\right\rangle\right)\left(\left\langle D_{[i][j]}^{2}\right\rangle /\left\langle D_{i j}^{2}\right\rangle\right),
$$

with $D_{i j}$ and $\bar{D}_{i j}$ according to Eqs. (15) and (37), respectively. For isotropic turbulence, we know [10, Eq. (3-20)] that

$$
\frac{\left\langle D_{[i][j]}^{2}\right\rangle}{\left\langle D_{i j}^{2}\right\rangle}= \begin{cases}2 / 15, & i=j \\ 1 / 10, & i \neq j\end{cases}
$$

The first factor in Eq. (39) can be calculated in the same manner as the other constants in this section. This correction factor gives a production term $\boldsymbol{P}$ to which all strain components in their time mcan value contribute with the same weight as they do in isotropic turbulence, regardless of the grid's anisotropy. This feature has been found to be important.

We get a "quasi-steady-state" model if we neglect the acceleration, convection, and diffusion terms in Eq. (35):

$$
V_{\overline{E^{\prime}}}=\left(c_{4} / c_{3}\right) h^{2 i j} c \bar{D}_{i j}^{2} .
$$

This is essentially the model used by Deardorff [4], except for the correction factor ${ }^{i j} c$ and the use of fluctuating velocities to compute $\bar{D}_{i j}^{2}$. It has been found 
that the replacement of Eq. (35) by Eq. (41) does not change the results considerably. This is quite a fortunate result because it shows that we do not need such complicated SGS transport equations as proposed in [7].

\section{Boundary Conditions and InItIal Values}

The exact wall boundary condition can be taken over directly for the normal velocity only:

$$
{ }^{3-}-\left._{3}\right|_{\text {wall }}=0
$$

For all the other variables we must use a model, since they are not defined as surface mean values taken at the wall. Even Eq. (42) would be an approximation if filtered velocities were used. Starting from the averaged basic equation, Eq. (9), we see that we need boundary conditions for the momentum flux due to the normal diffusion only. For example,

$$
\left.\tau_{w} \equiv \nu \frac{3 \overline{\partial u_{1}}}{\partial x_{3}}\right|_{\text {wall }}
$$

For this we assume

$$
\tau_{w}=\left.\left.\frac{1 \overline{u_{1}}}{\left\langle\overline{u_{1}}\right\rangle}\right|_{1}\left\langle\tau_{w}\right\rangle\right|_{\mathrm{wall}}
$$

The subscript 1 corresponds to the grid-index $n$ in Fig. 3 . For the time mean value of $u_{1}$ we assume the law of the wall in the following way.

$$
\left.\left\langle\overline{u_{1}}\right\rangle\right|_{1}=\frac{\left\langle\tau_{w}\right\rangle^{1 / 2}}{\kappa}\left[\ln \left(\left.\frac{\Delta x_{3}}{2}\right|_{1} E\right)-1\right] .
$$

Here, $E$ accounts for the wall roughness at the wall. We use the same value as Deardorff [4], $E=8.8 \times 10^{4} / D$. The term -1 results from averaging the logarithmic law over the wall-adjacent mesh. The time-mean wall stress at the wall is known a priori, for a plane channel with equal wall properties at both walls from an integral momentum balance, to be equal to unity. For an annulus we use Eq. (45) 
to calculate $\left\langle\tau_{w}\right\rangle$ for that value of $\left.\left\langle{ }^{1} \bar{u}_{1}\right\rangle\right|_{1}$ found as the average value of the velocities $\left.{ }^{1} \bar{u}_{1}\right|_{1}$ in the foregoing integration step. (This is different from Schumann [19], where no suitable method had been found.) For the velocity ${ }^{2} \bar{u}_{2}$ we take a boundary condition in which the normal flux is calculated assuming a linear velocity profile and a constant eddy viscosity within the wall-adjacent grid volumes.

The boundary condition for the kinetic energy is of the form

$$
\left.\frac{\partial\left(\bar{E}^{\prime}\right)}{\partial x_{3}}\right|_{\text {wall }}=\frac{\left.c_{11} V_{\overline{E^{\prime}}}\right|_{1}}{\left.\frac{\Delta x_{3}}{2}\right|_{1}}
$$

If $c_{11}$ is equal to unity, this condition implies a linear variation of ${ }^{V} \bar{E}^{\prime}$ near the wall. This seems to be inappropriate because the kinetic energy profile varies strongly and assumes a maximum value in the proximity of the wall, which might fall into the grid volume adjacent to the wall. Therefore, a smaller value of $c_{11}$ should be taken. On the other hand, as the diffusion is zero at the wall, in principle we might take $c_{11}=0$. But a positive value of $c_{11}$ allows us to account for the dissipation in the viscous sublayer. Without any further reasoning we assume $c_{11}=0.2$. Moreover, the eddy viscosity for ${ }^{V} \bar{E}^{\prime}$ at the wall is set equal to that value resulting for the middle of this grid volume.

Finally, boundary conditions are needed for the calculation of $\bar{D}_{i j}^{2}$ according to Eq. (37). Here we assume linear variations of the velocity fluctuations $\left({ }^{i} \bar{u}_{i}-\left\langle{ }^{i} \bar{u}_{i}\right\rangle\right)$ near the walls.

No artificial boundary conditions are needed for the convective terms. These all cancel out due to Eq. (42) at the wall. Equations (44) and (45) are consistent with the known law of the wall and are applicable even for rather coarse grid systems. No isotropy condition is used here as it was in Deardorff [4].

For the $x_{1}$ - and $x_{2}$-directions, we assume periodicity as boundary conditions with periodic lengths $X_{1}$ and $X_{2}$, the values of which are given in Table Il. These rather artificial conditions can be defended if the periodic lengths are at least twice the length over which the field variables are correlated noticeably. From the experiments of Comte-Bellot [3], we conclude that the values used (see Table II) are large enough (except for cases $K 1, Z 1$ ).

The initial velocities are calculated out of two potentials (to assure continuity) which are established using random numbers with a prescribed energy spectrum according to Eq. (12). The initial values of SGS kinetic energy are determined afterward from Eq. (41).

Starting from these initial values the variables are stepped forward by the leap-frog method [4]. After about two dimensionless time units the solutions have reached a steady state in their statistical values, though remaining time- and spacedependent for all time in their local values. 


\section{Numerical Verification of the Method}

Two channel flows are considered: one plane channel (labeled " $K n$ ") and the flow in an annulus with the ratio of external to internal radius of $5: 1$ (" $Z n$ "). Both cases were simulated with four $(n=1,2,3,4)$ different grid systems, each having different numbers of grid volumes and periodic lengths. The values used are shown in Table II. The largest grid, with 65,536 volumes, is used in cases $K 4$ and $Z 4$. The number of grid volumes is considerably larger than that used by Deardorff [4], who used 6720 volumes. In cases $K 4$ and $Z 4$, nonequidistant mesh

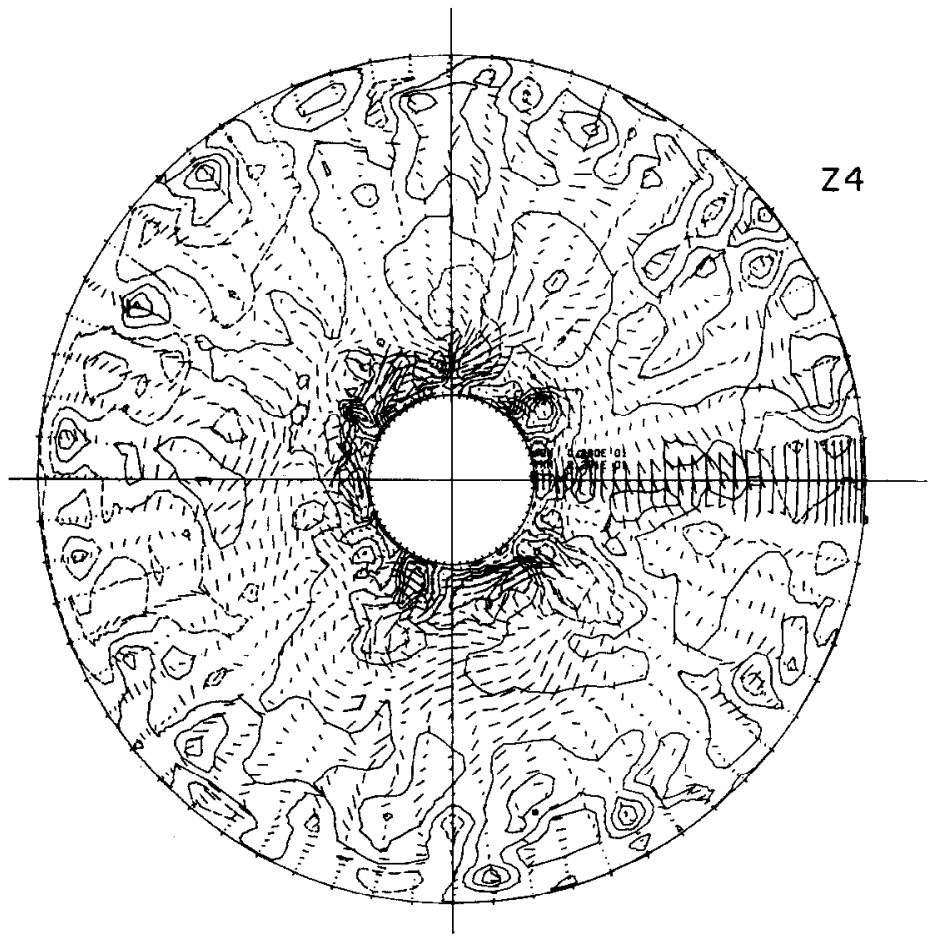

FIG. 4. Fluctuating velocities at one time in an annulus with the ratio of radii of 5:1. The velocity components in the plane of the drawing are represented by the vectors; the axial fluctuating velocities are represented by the contour lines.

spacings are used in the cross-stream direction. Moreover, in these cases the initial conditions are evaluated out of the final results found for $K 3$ and $Z 3$ by linear interpolation. In case $K 3$ the grid side lengths are equal, whereas they are very much different in case $K 4$. So comparison of the results for cases $K 3$ and $K 4$ 


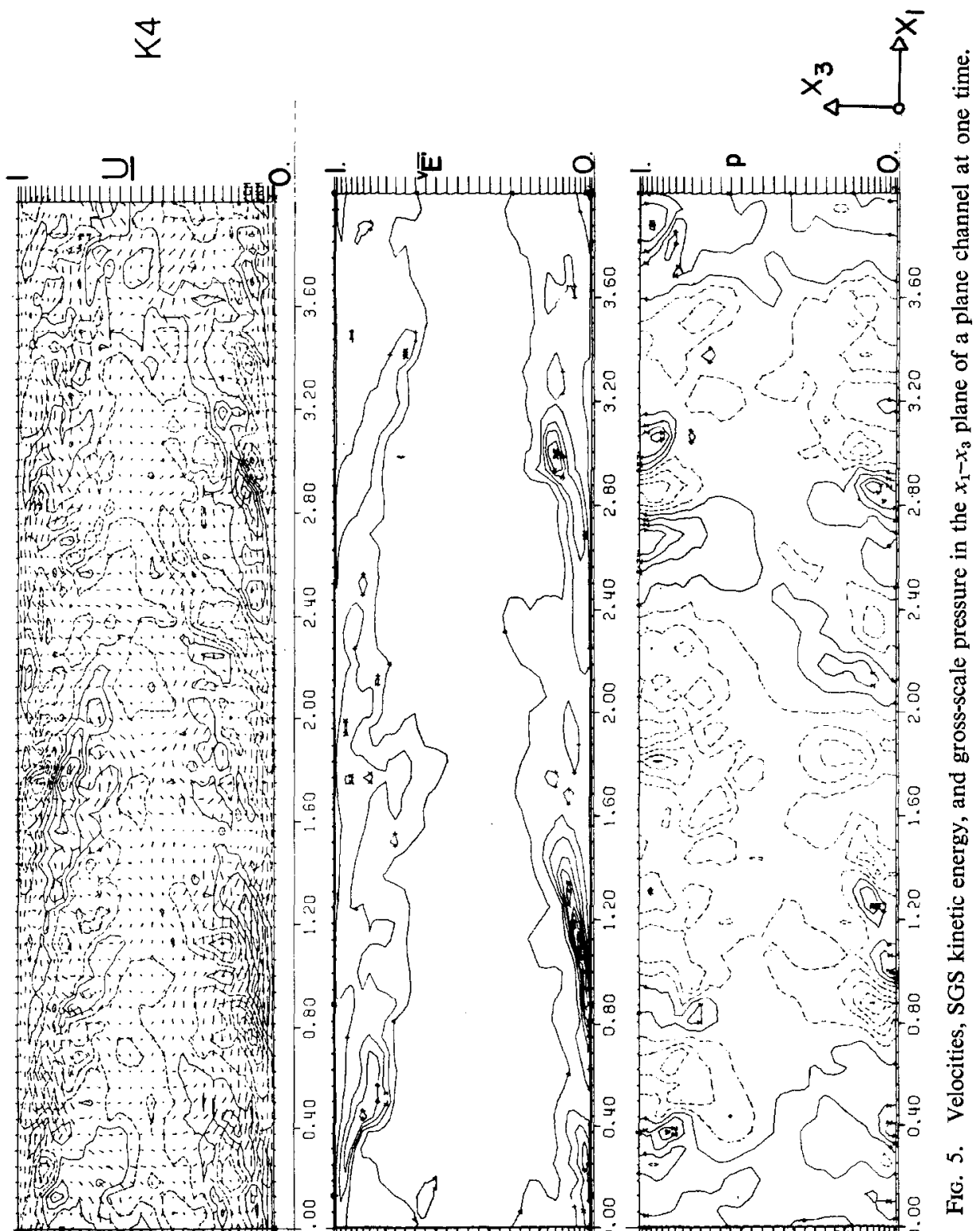


is a good test for the ability of the present theory to account for anisotropic grids.

In the following, results are reported for different realizations of the flows. Those labeled $K n$ and $Z n$ are preliminary results reported already in [19, 20]. In that study the steady-state solution had not been reached for cases 3 and 4 . Since then, some further integrating has been performed for cases $K 3, K 4$, and $Z 3$ using the revised method described here. Moreover, in addition to averaging over planes parallel to walls, the results found for some specific times [21] have been averaged in order to reduce the statistical fluctuations. These results are denoted by $K 3+, K 4+, Z 3+$. The Reynolds number is $\mathrm{Re}_{m}=3 \times 10^{5}$.

Figures 4 and 5 show some typical flow patterns. Displays are shown of the instantaneous fluctuating velocities, the SGS kinetic energy ${ }^{\prime} \bar{E}$, and the gross scale pressure.

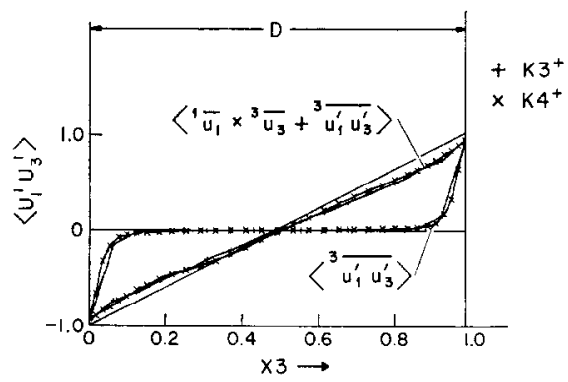

Fig. 6. Time mean values of the SGS and the total Reynolds stresses. The inclined straight line gives the steady-state value of the total stresses.

The influence of the SGS theory can be seen from Fig. 6, which shows the time mean values of the total Reynolds stress $\left\langle u_{1}{ }^{\prime} u_{3}{ }^{\prime}\right\rangle$ for cases $K 3+$ and $K 4+$. We see that $\left\langle\overline{u_{1}{ }^{\prime} u_{3}{ }^{\prime}}\right\rangle$, which is that part of the stress predicted by the SGS model, is negligible over the range $0.1 \leqslant x_{3} \leqslant 0.9$, but of large importance near the walls. The fact that the numerical results for the stress do not coincide with the theoretical line shows that the steady state is still not reached completely. It should be noted that the SGS stress seen in this figure is solely due to the "inhomogeneous" part of the SGS model, Eq. (16), whereas the "isotropic" part mainly determines the SGS kinetic energy. The SGS model contributes up to $40 \%$ of the kinetic energy (see Fig. 8).

Table III shows the computed maximum velocity values compared with some experimental values. Agreement seems to be satisfactory for the plane channel even for rather coarse grids. Barthels [1] and Maubach [16] found the maximum velocity value for the annulus to be $1 \%-2 \%$ lower than for a plane channel. Such small variations lie within the statistical errors of the numerical results. But we find that the tendency is described correctly. 
TABLE III

Maximum Velocity

\begin{tabular}{|c|c|c|c|}
\hline$\left\langle u_{1}\right\rangle_{\max }$ & $R 2 / R 1$ & $10^{-5} \cdot \operatorname{Re}_{m}$ & Reference \\
\hline \multicolumn{4}{|c|}{ Plane channel } \\
\hline 31.8 & 1 & 1 & Clark [2] \\
\hline 31.5 & 1 & 4.6 & Comte-Bellot [3] \\
\hline 27.7 & 1 & 2.4 & Comte-Bellot [3] \\
\hline 27 & 1 & 1 & Laufer [12] \\
\hline 30.1 & 1 & $\infty$ & Deardorff [4] \\
\hline 28.9 & 1 & 3 & $K 1$ \\
\hline 31.3 & 1 & 3 & $K 2$ \\
\hline 27.4 & 1 & 3 & $K 3$ \\
\hline 28.2 & 1 & 3 & $K 3+$ \\
\hline 27.6 & 1 & 3 & $K 4$ \\
\hline 28.3 & 1 & 3 & $K 4+$ \\
\hline \multicolumn{4}{|c|}{ Annulus } \\
\hline 28.0 & 5 & 3 & $Z 1$ \\
\hline 28.3 & 5 & 3 & $Z 2$ \\
\hline 27.6 & 5 & 3 & $Z 3$ \\
\hline 28.6 & 5 & 3 & $Z 3+$ \\
\hline 27.9 & 5 & 3 & $Z 4$ \\
\hline
\end{tabular}

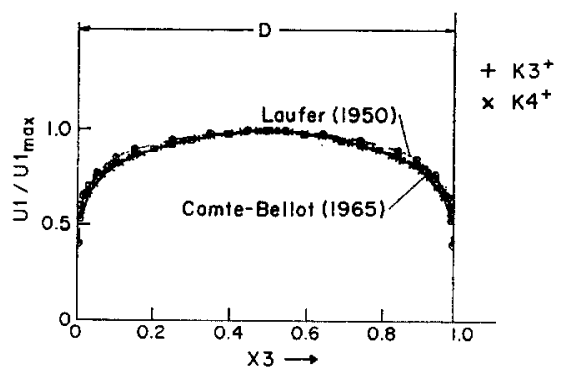

FIG. 7. Normalized velocity profile in comparison with measured values (dashed curves). 


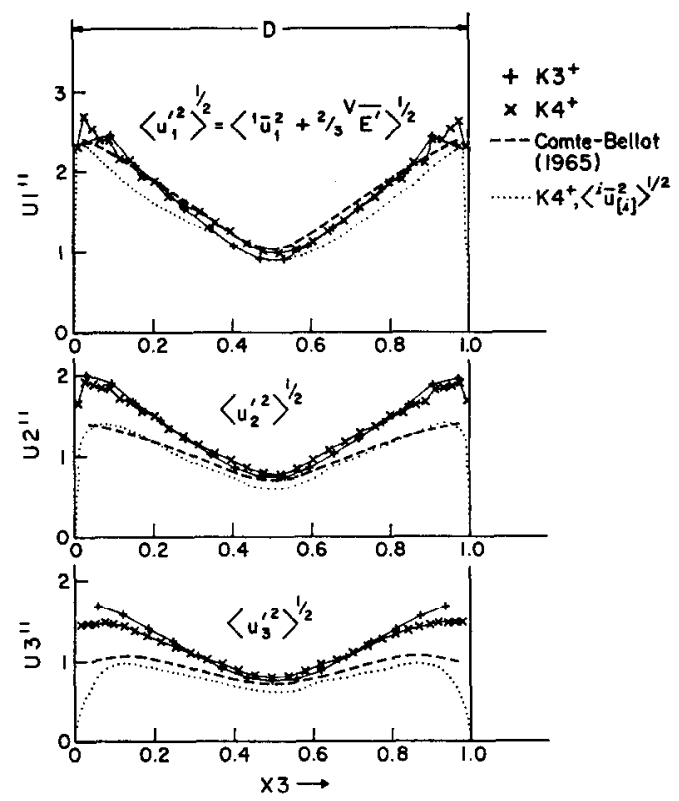

FIG. 8. Root mean square values of the fluctuating velocity components in comparison with measured values (dashed curves). The dotted curves show the contribution by the gross scale motion.

The mean axial velocity profile is shown in Fig. 7 in comparison with the values measured by Laufer [12] and Comte-Bellot [3]. Especially the later ones agree well with the numerical results. A sensitive measure of the profile is the difference between its maximum and mean values. The results obtained for this difference are reported in Table IV. Here we see that agreement between the calculated and the experimental values is satisfactory and that it is better than for the results obtained by Deardorff [4]. This seems to be a consequence of the new SGS theory.

A similar improvement can be recognized in the profiles of the rms values of the fluctuating velocities as shown in Fig. 8, where they are compared with the values measured by Comte-Bellot [3]. The agreement is very good for the axial component and generally in the middle of the channel, whereas there is some departure in the other components near the walls. We have to keep in mind, however, that differences between the experimental results obtained by different workers $[2,3,12]$ for these quantities are of about the same magnitude. As a whole, the agreement is considerably better than for the results of Deardorff [4]. The same has been found for case $K 1$ and $K 2[19,20]$.

Principal agreement was established between the measured values of ComteBellot [3], the numerical results of Deardorff [4], and this approach concerning 


\section{TABLE IV}

Difference between Maximum and Mean Velocity

\begin{tabular}{cccl}
\hline$\left\langle u_{1}\right\rangle_{\max }-u_{1 m}$ & $R 2 / R 1$ & $\mathrm{Re}_{m} \cdot 10^{-5}$ & \multicolumn{1}{c}{ Reference } \\
\hline 2.32 & 1 & 1 & Clark [2] \\
2.70 & 1 & 3 & Barthels [1] \\
2.68 & 5 & 3 & Barthels [1] \\
5 & 1 & $\infty$ & Deardorff [4] \\
3.19 & 1 & 3 & $K 1$ \\
3.17 & 1 & 3 & $K 2$ \\
2.60 & 1 & 3 & $K 3$ \\
3.08 & 1 & 3 & $K 4$ \\
2.87 & 1 & 3 & $K 4+$ \\
3.14 & 1 & 3 & $Z 1$ \\
2.75 & 5 & 3 & $Z 2$ \\
2.52 & 5 & 3 & $Z 3$ \\
2.74 & 5 & 3 & $Z 4$ \\
3.28 & 5 & 3 & \\
3.15 & 5 & 3 & \\
\hline
\end{tabular}

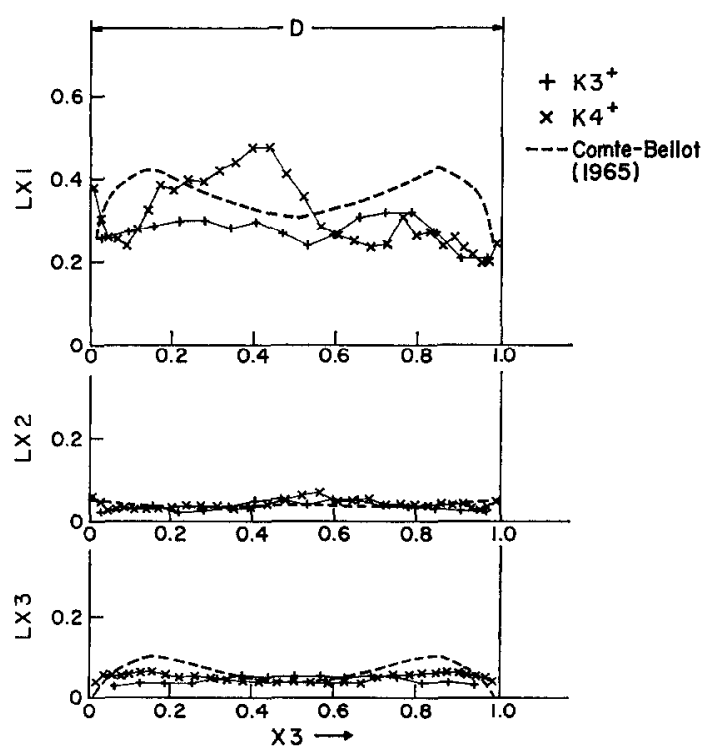

FIG. 9. Axial integral correlation lengths for the different velocity components in comparison with measured values. These figures are unsmoothed computer drawings. The large fluctuations are due to the small number of realizations over which the averages have been taken. 
the axial integral correlation lengths for the three different velocity components. Figure 9 shows the numerical results of

$$
\left.L X_{i} \equiv \int_{0}^{x_{1} / 2}\left\langle\stackrel{\leftarrow}{u}_{[i]}^{n}\left(x_{1}, x_{2}, x_{3}\right) \cdot \stackrel{i-u}{u}_{[i]}^{\prime \prime}\left(x_{1}+z_{1}, x_{2}, x_{3}\right)\right\rangle d z_{1}\left\langle\stackrel{i}{i} \stackrel{\sim}{[i]}^{2}\right\rangle\right\rangle^{-1}
$$

in comparison with those values measured by Comte-Bellot [3]. The double primes denote the departure of time mean. Although there are still large statistical fluctuations we see that the correlation length of the axial velocity fluctuation is about five times that of the other velocity fluctuations. Corresponding results have been found for the annulus $[19,21]$.

\section{CONCLUSIONS}

A method has been developed for direct numerical simulation of incompressible turbulent channel flows in a plane channel and an annulus. A SGS theory is used which accounts for the inhomogeneities of the turbulence and the anisotropy of the finite difference grid and the finite difference operators. The results are in good agreement with experimental data. This agreement is virtually independent of the number of grid cells employed. Moreover, we get the same results for different degrees in the anisotropy of the grid. This seems to be a strong indication of the usefulness of the SGS theory.

The most important differences between the present method and older ones, especially that of Deardorff [4], are: (a) splitting of the SGS stresses into inhomogeneous and locally isotropic parts; (b) accounting for the anisotropy of the finite difference scheme; (c) appropriate boundary conditions; (d) higher grid resolution; (e) use of a SGS kinetic energy transport equation; (f) larger values for the periodicity lengths. These differences are listed in the order of their estimated importance.

The most important limitations of the method are: (a) The method is restricted to high Reynolds numbers; (b) the method is not completely independent of empirical information; (c) large amounts of computer time are required if flow details are desired (refer to Table II). Moreover, although much has been done to account for unequal-sided grid cells we must be aware of the problematic nature of using cylindrical coordinates. The method of using cylindrical coordinates for the simulation of pipe flow cannot be recommended, for example, since it would resolve nearly all scales of turbulence near the center line but only a small range of scales near the walls. It is doubtful whether the different correction factors proposed are valid for such a large variation.

The method has been used successfully to investigate turbulence closure 
assumptions used in empirical models. It is possible to evaluate some of the constants appearing in them, especially for those terms which are mainly determined by the gross scale structure of the turbulent flows and are zero in isotropic turbulence. These results have been reported in [20]. They showed that some of the empirical "constants" used in simple turbulence models are dependent of the cross-stream position. In [21] it has been shown that the present method can be used to calculate wall-pressure correlation functions and the resultant forces acting on the inner cylinder of the annulus.

\section{APPENDIX: Calculation of Volume-Averaged Velocity Correlations}

We assume $u_{\alpha}(\mathbf{x}, t)$ and $u_{\beta}(\mathbf{x}, t)$ to be any two components of an isotropic velocity field having the two-point correlation

$$
R_{i j}(\mathbf{r}) \equiv\left\langle u_{i}(\mathbf{x}) u_{j}(\mathbf{x}+\mathbf{r})\right\rangle, \quad i, j=1,2,3 .
$$

For turbulence having Kolmogorov's spectrum this is [10,19]

$$
R_{i j}(\mathbf{r})= \begin{cases}\left.-f_{2}\langle\epsilon\rangle^{2 / 3} r^{2 / 3}\left[1-r_{[i]}^{2}\right]\left(4 r^{2}\right)\right]+R_{i j}(\mathbf{0}), & i=j, \\ -f_{2}\langle\epsilon\rangle^{2 / 3} r_{i} r_{j} /\left(4 r^{4 / 3}\right), & i \neq j,\end{cases}
$$

where

$$
r=\left(r_{i} r_{i}\right)^{1 / 2}, \quad f_{2}=(18 / 55) \alpha \Gamma(1 / 3) .
$$

We want to calculate correlations of type

$$
R\left(u_{\alpha}, u_{\beta}, V_{\alpha}, V_{\beta}, \xi\right) \equiv\left\langle{ }^{V} \overline{u_{\alpha}}(\mathbf{x}){ }^{V_{\beta}} \overline{u_{\beta}}(\mathbf{x}+\xi)\right\rangle .
$$

The prescripted overbars denote averages taken over space volumes $V_{\alpha, \beta}$. These averages can be formulated (e.g., for subscript $\alpha$ ) [23]:

$$
V_{\alpha} \overline{u_{\alpha}}=\iiint_{\infty} K_{\alpha}(\mathbf{y}) u_{\alpha}(\mathbf{y}) d V(\mathbf{y})
$$

with

$$
K_{\alpha}(\mathbf{y})= \begin{cases}1 / V_{\alpha}, & \mathbf{y} \in V_{\alpha}, \\ 0, & \mathbf{y} \notin V_{\alpha} .\end{cases}
$$

We start from the definitions

$$
R\left(u_{\alpha}, u_{\beta}, V_{\alpha}, V_{\beta}, \xi\right)=\left\langle\iint_{\infty} K_{\alpha}(\mathbf{y}) u_{\alpha}(\mathbf{y}) d V(\mathbf{y}) \cdot K_{\beta}(\mathbf{z}) u_{\beta}(\mathbf{z}+\xi) d V(\mathbf{z})\right\rangle .
$$


The integration involving time averaging and the two integrations with respect to $\mathbf{y}$ and $\mathbf{z}$ can be interchanged; let $\tau=\mathbf{z}-\mathbf{y}, d V(\tau)=d V(\mathbf{z})$; then

$$
R\left(u_{\alpha}, u_{\beta}, V_{\alpha}, V_{\beta}, \xi\right)=\iiint_{\infty} \phi_{\alpha \beta}(\tau) R_{\alpha \beta}(\tau-\xi) d V(\tau)
$$

Here, $\phi_{\alpha \beta}$ is the "volume-correlation,"

$$
\phi_{\alpha \beta}(\tau) \equiv \iiint_{\infty} K_{\alpha}(\mathbf{y}) K_{B}(\tau+\mathbf{y}) d V(\mathbf{y}),
$$

which can be evaluated analytically for any rectangular volume $V_{\alpha}, V_{\beta}$.

In this study we need this volume-correlation for two types of pairs of volumes:

(a) $V_{\alpha}=V_{\beta}=V_{(n)}$, where $\cdot V_{(n)}$ is an $n$-dimensional volume, which means it is either a line $(n=1)$ of length $H_{1}$ or a plane $(n=2)$ of side lengths $H_{1}$ and $H_{2}$ or a real volume $(n=3)$ of side lengths $H_{1}, H_{2}, H_{3}$. For these volumes Eq. (A-8) gives

$$
\phi_{\alpha \beta}(\tau)=\prod_{i=1}^{n} \frac{1}{H_{[i]}^{2}} \max \left(0, H_{i}-\left|\tau_{i}\right|\right) .
$$

(b) $\quad V_{\alpha}$ and $V_{\beta}$ are two finite planes which are perpendicular to each other with coinciding centers of gravity (see Fig. 10):

$$
\begin{aligned}
& V_{\beta}:\left\{\mathbf{y} ;\left|y_{1}\right| \leqslant H_{1} / 2, y_{2}=0,\left|y_{3}\right| \leqslant H_{3} / 2\right\}, \\
& V_{\alpha}:\left\{\mathbf{y} ; y_{1}=0,\left|y_{2}\right| \leqslant H_{2} / 2,\left|y_{3}\right| \leqslant H_{3} / 2\right\} .
\end{aligned}
$$

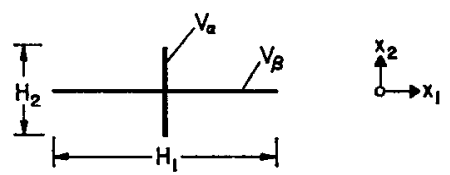

FIG. 10. Configuration of planes over which averages are taken.

Here we find

$$
\phi_{\alpha \beta}(\tau)=\left\{\begin{array}{l}
\frac{1}{H_{1} H_{2} H_{3}^{2}} \max \left(0, H_{3}-\left|\tau_{3}\right|\right), \\
\text { if }\left(\left|\tau_{1}\right| \leqslant H_{1} / 2\right) \text { and }\left(\left|\tau_{2}\right| \leqslant H_{2} / 2\right), \\
0, \quad \text { elsewhere. }
\end{array}\right.
$$

Using these tools, all volume-averaged velocity correlations needed can be evaluated. This shall be shown for two examples. 
(a) We ask for that portion of SGS-kinetic energy contained within a line, a plane, or a volume:

$$
\left\langle{ }^{V(n)} \overline{E^{\prime}}\right\rangle \equiv \frac{1}{2}\left\langle\left(u_{i}-\frac{V(n)-\bar{u}}{u_{i}}\right)^{2}\right\rangle
$$

We find

$$
\begin{aligned}
\left\langle{ }^{V(n)} \overline{E^{\prime}}\right\rangle & =\frac{1}{2}\left[\left\langle^{V(n)} \overline{u_{i}^{2}}\right\rangle-\left\langle\left({ }^{V(n)} \overline{u_{i}}\right)^{2}\right\rangle\right] \\
& =\frac{1}{2} \sum_{i=1}^{3}\left[R_{i i}(\mathbf{0})-R\left(u_{i}, u_{i}, V_{(n)}, V_{(n)}, 0\right)\right] \\
& =f_{1}\langle\epsilon\rangle^{2 / 3} h^{2 / 3} 2^{n} \int_{0}^{H_{1} / h} \cdots \int_{0}^{H_{n} / h}\left(\prod_{j=1}^{n}\left(H_{j}-\tau_{j}\right)\right)\left(\tau^{2}\right)^{1 / 3} d \tau_{1} \cdots d \tau_{n},
\end{aligned}
$$

where

$$
f_{1}=\alpha \Gamma(1 / 3)(9 / 20) ; \quad h=V_{(n)}^{1 / n} .
$$

For $n=1$ the integral is equal to $9 / 40$. For $n>1$ the integral must be evaluated numerically. The results can be approximated with an error of about $1 \%$ by

$$
\begin{aligned}
\left\langle{ }^{V(n)} \overline{E^{\prime}}\right\rangle & =f_{1}\langle\epsilon\rangle^{2 / 3} h^{2 / 3} \frac{9}{20}\left(\sum_{i=1}^{n}\left(\frac{H_{i}}{h}\right)^{1 / \beta}\right)^{2 \beta / 3}, \\
\beta & =0.69687 .
\end{aligned}
$$

(b) As"an example for the time mean value of finite difference form of the squared strain tensor we consider $\left\langle\bar{D}_{12}^{2}\right\rangle$ as appears in Eq. (25):

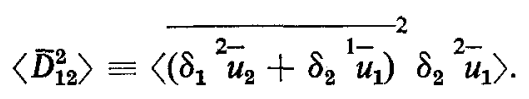

Using the definitions of the finite difference and the algebraic averaging operators we find for isotropic turbulence:

$$
\begin{aligned}
\left\langle\bar{D}_{12}^{2}\right\rangle= & \frac{1}{\Delta x_{2}{ }^{2}}\left\{\left\langle{ }^{1} \bar{u}_{1}{ }^{2} \bar{u}_{2}\left(0, \Delta x_{2} / 2,0\right)\right\rangle-\left\langle\bar{u}_{1}{ }^{2-}{ }_{1}\left(0,3 / 2 \Delta x_{2}, 0\right)\right\rangle\right\} \\
& -\frac{2}{\Delta x_{1} \Delta x_{2}}\left\langle\left\langle\bar{u}_{1}{ }^{2-} \bar{u}_{2}\left(\Delta x_{1} / 2, \Delta x_{2}, 0\right)\right\rangle .\right.
\end{aligned}
$$

Here the parameters behind velocity products specify the components of the 
distance vector between the centers of gravity of the different finite planes over which the averages are taken. We see that we have to evaluate, e.g.,

$$
\left\langle\bar{u}_{1}{ }^{2} \bar{u}_{2}\left(\xi_{1}, \xi_{2}, 0\right)\right\rangle=R\left(u_{1}, u_{2},{ }^{1} F,{ }^{2} F,\left\{\xi_{1}, \xi_{2}, 0\right\}\right) \text {. }
$$

The volume correlation is that given by Eq. (A-10) with $H_{i}=\Delta x_{i}$. The result is $\left\langle\bar{u}_{1}{ }^{2-} \bar{u}_{2}\left(\xi_{1}, \xi_{2}, 0\right)\right\rangle=-f_{2}\langle\epsilon\rangle^{2 / 3} h^{2 / 3}$

$\times \frac{1}{2} \int_{-h_{1} / 2}^{h_{1} / 2} \int_{-h_{2} / 2}^{h_{2} / 2} \int_{0}^{h_{3}} \frac{h_{3}-\tau_{3}}{h_{3}} \frac{\left(\tau_{1}+z_{1}\right)\left(\tau_{2}+z_{2}\right)}{\left[\left(\tau_{1}+z_{1}\right)^{2}+\left(\tau_{2}+z_{2}\right)^{2}+\tau_{3}{ }^{2}\right]^{2 / 3}} d \tau_{3} d \tau_{2} d \tau_{1}$,

where

$$
h_{i}=\Delta x_{i} / h ; \quad z_{i}=\xi_{i} / h ; \quad h=\left(\Delta x_{1} \Delta x_{2} \Delta x_{3}\right)^{1 / 3} .
$$

This integral (and similar ones for the other terms in Eqs. (A-15) and (25)) must be evaluated numerically. Some approximate algebraic equations for these integrals are listed in [19].

We see that the method is capable of evaluation of all volume-averaged correlations needed. The only assumptions are isotropy and Kolmogorov's spectrum.

\section{Summary of Operator Notation}

$\begin{array}{ll}y & \text { Any (dimensionless) quantity } \\ y & \text { Dimensional quantity } \\ \mathbf{y} \equiv\left\{y_{1}, y_{2}, y_{3}\right\} & \text { Vector } \\ \langle y\rangle & \text { Time mean value } \\ y^{\prime \prime} \equiv y-\langle y\rangle & \text { Departure of time mean value } \\ v_{\bar{y}} & \text { Volume mean value; see Eq. (5) and Fig. } 2 \\ v^{i} \bar{y} & \text { Volume mean value; the volume is shifted in the } i \text {-direction } \\ & \text { by } \Delta x_{i} / 2 ; \text { see Fig. } 2 \\ { }^{i} \bar{y} & \text { Surface mean value; the normal of the surface }\left(^{i} F\right) \text { is in the } \\ & i \text {-direction; see Eq. (6) and Fig. } 2 \\ y^{\prime} & \text { Departure of the mean value used in the context } \\ \delta_{i} y & \text { Finite differential in } i \text {-direction; see Eq. (7) } \\ \bar{y}^{i} & \text { Algebraic mean value of } y \text { at two positions separated by one } \\ & \text { grid interval in } i \text {-direction } \\ y_{m} & \text { Mean value taken over the channel cross section } \\ y_{\max } & \text { Maximum value }\end{array}$




\section{REFERENCES}

1. H. Barthels, Darstellung des Wärmeüberganges in konzentrischen Ringspalten unter Benulzung der Analogie zwischen Impuls- und Wärmeaustausch, Jül-506-RB (1967).

2. J. A. Clark, J. Basic Eng. (Tr. ASME) 90 (1968), 455-467.

3. G. Comte-Bellor, Écoulement Turbulent Entre Deux Parois Parallèles, Publ. Sci. et Techn. du Ministère de l'Air No. 419 (1965).

4. J. W. DeardorfF, J. Fluid Mech. 41 (1970), 453-480.

5. J. W. Deardorff, J. Comp. Phys. 7 (1971), 120-133.

6. J. W. Deardorff, J. Atmos. Sci. 29 (1972), 91-115.

7. J. W. DeardorfF, J. Fluids Eng. (Tr. ASME) (1973), 429-438.

8. E. R. van Driest, J. Aeron. Sci. 23 (1956), 1007.

9. F. M. Galloway and R. J. Adler, J. Comp. Phys. 10 (1972), 379-399.

10. J. O. Hinze, "Turbulence," McGraw Hill, New York, 1959.

11. A. N. Kolmogorov, Die lokale Struktur der Turbulenz in einer zähen kompressiblen Flüssigkeit (1941), German transl. in "Sammelband zur Statistischen Theorie der Turbulenz" (H. Göring, Ed.), Akademie-Verlag, Berlin, 1958.

12. J. LAUfER, Investigation of Turbulent Flow in a Two-Dimensional Channel, NACA Report $1053(1950)$.

13. A. Leonard, Advan. Geophysics 18A (1974), 237-248.

14. D. K. LlLLY, On the Application of the Eddy Viscosity Concept in the Inertial Sub-range of Turbulence, NCAR Manuscript No. 123 (1966).

15. D. K. LILLY, The Representation of Small-Scale Turbulence in Numerical Simulation Experiments, in "Proceedings of the IBM Scientific Computing Symposium on Environmental Sciences” (H. H. Goldstine, Ed.), pp. 195-210, IBM Form No. 320-1951 (1967).

16. K. Maubach, Chemie Ing. Tech. 42 (1970), 995-1003.

17. J. C. RotTA, "Turbulente Strömungen," B. G. Teubner, Stuttgart, 1972.

18. W. SCHÖNAUER, Die Lösung der dreidimensionalen instationären Navier-Stokes-Gleichung mit dem Differenzenverfahren, DFVLR-report DLR FB 70-15 (1970).

19. U. Schumann, Ein Verfahren zur direkten numerischen Simulation turbulenter Strömungen in Platten- und Ringspaltkanälen und über seine Anwendung zur Untersuchung von Turbulenzmodellen, Dissertation TH Karlsruhe, KFK 1854 (1973). Summarizing English transl. by M. Love, Queen Mary College, University of London, 1974.

20. U. Schumann, Results of a Numerical Simulation of Turbulent Channel Flows, in "Proceedings of the ANS International Meeting on Reactor Heat Transfer," Karlsruhe, October 1973 (M. Dalle-Donne, Ed.), Paper No. 40.

21. U. SchumanN, Nucl. Eng. Design 32 (1975), 37-46.

22. J. S. SMAGurinsky, Monthly Weather Rev. 91 (1963), 99-164.

23. M. S. Uberoi AND L. S. G. Kovasznay, Quart. Appl. Math. 10 (1952), 375-393.

24. G. P. Williams, J. Fluid Mech. 37 (1969), 727-750. 